\section{Zauberworte: Kopfpauschale und Bürgerversicherung}

Schon jetzt ist abzusehen, dass die „Gesundheitsreform im Miniformat" nicht lange halten wird. Die Bürgerverunsicherung wird weitergehen. Die neuen Zauberworte sind Bürgerversicherung und Kopfpauschale.

In die Bürgerversicherung würden Beamte und Selbstständige mit einbezogen. Zinserträge, Mieteinnahmen und Aktiengewinne sollen für die Beiträge mit herangezogen werden. Der Hintergedanke ist, das Sozialsystem vom Faktor Arbeit zu entkoppeln. So soll verhindert werden, dass die Beiträge in Zeiten hoher Arbeitslosigkeit aus dem Ruder laufen. Vorhersehbar ist ein gewaltiger bürokratischer Aufwand, um für jede Person insbesondere bei wechselndem Einkommen den Beitrag zu berechnen.

Für besser hielte ich persönlich eine Kopfpauschale: Jeder Erwachsene hätte einen einheitlichen festen Beitrag zu zahlen, unabhängig vom Einkommen. Der monatliche Beitrag kann so gestaltet werden, dass eine zu definierende
Basisleistung für jeden garantiert ist und durch Zusatzmodule - ähnlich den Autoversicherungen - Zusatzleistungen eingekauft werden können. Wenn jemand erster Klasse nach Paris oder New York fliegen möchte, und es sich auch leisten will, dann lasst ihn doch!

Kommen dann noch ein Selbstverantwortungsfaktor und eine Rücklage für steigende Kosten im Alter hinzu, dann wäre eine Strukturreform zu schaffen, die besser und sogar viel länger als nur ein paar Jahre funktionieren würde. Dr. Werner Kersten, Moers

\title{
Kongress mit buntem Rahmen
}

\section{Ein Forum für Allergien parallel als Publikumsmesse und als interdisziplinärer Fachkongress - mit diesem Konzept war die Allergica 2002 erstmals angetreten. Ihre zweite Auflage erlebt sie eingebettet in das Forum chronische Krankheiten von 14. bis 16. November 2003 auf dem Frankfurter Messegelände.}

$\mathrm{n}$ die Allergica integriert ist auch dieses Jahr ein medizinischer Fachkongress, der von einem hochrangigen Advisory-Board begleitet wird und mit der Höchstpunktzahl von 18 Punkten zertifiziert wurde. Hier erhalten Hausärzte, Apotheker und Fachpersonal wertvolle Hinweise, die sich schnell in die Praxis umsetzen lassen. So werden unter anderem Kurse unter der Leitung von folgenden Referenten angeboten:

—Dr. Horst Müsken (Allergie- und

Asthma-Klinik Bad Lippspringe): In-vitro- und In-vivo-Diagnostik

— Prof. Dr. Karl-Christian Bergmann

(Allergie- und Asthma-Klinik Bad Lippspringe): nasale, bronchiale und orale Provokation

— Dr. Josef Lecheler (CJD Asthmazentrum Berchtesgaden): Disease-Management-Programme in der Praxis: Asthma

Außerdem finden Symposien statt, die Themen wie atopische Dermatitis oder Asthma bei Säuglingen und Kleinkindern aus der Sicht der verschiedenen Fachärzte (Dermatologen, Hausärzte, Kinderärzte, Pneumologen etc.) beleuchten. Vorgestellt werden verschie-

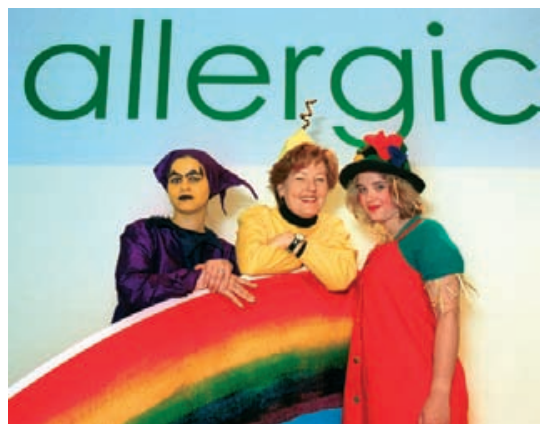

Impressionen von der Allergica 2002: Kindertheater bei allergica4kids mit den Figuren Allergikus, Sputz und Karline Reibutz (Foto oben) und praktische Übungen beim Seminar „Der allergologische Notfall in der Praxis" mit Prof. Dr. Günther Forck (Foto unten).

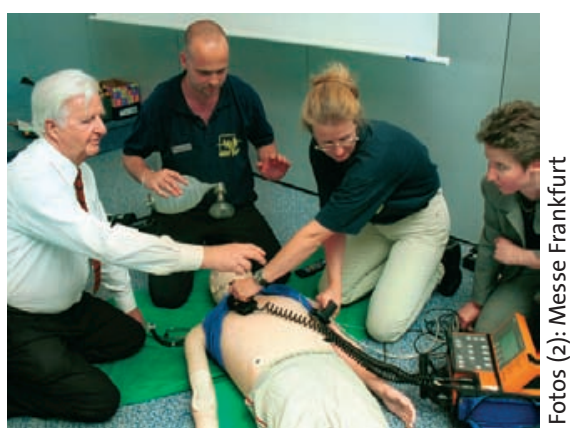

dene Therapie-, Rehabilitations- und Behandlungsmaßnahmen, die Anlass geben für den Austausch unter Kollegen.

Tipp: Nutzen Sie den FrühbucherRabatt und melden Sie sich rechtzeitig online an unter allergica.com oder dialogdiabetes.com.

Als Fach- und Publikumsveranstaltung bietet der Kongress auch ein buntes Rahmenprogramm für Betroffene und Interessierte wie beispielsweise Schnupperschulungen zum Thema Allergien, Vorträge und eine Mess-Strasse, in der sich Besucher selbst durchchecken können. Für Kinder gibt es bei Medilino4kids, einen extra Messeparcours mit verschiedenen WorkshopStationen, wo die jungen Besucher unter anderem ihr Lungenvolumen testen und ihre Fußreflexzonen kennenlernen können.

\section{Prof. Dr. Ludger Klimek, Wiesbaden}

Impressum „Der ÄDA informiert"

Verantwortlich für den Inhalt: Prof. Dr. Ludger Klimek, Wiesbaden Redaktion: Markus Seidl

Urban \& Vogel Medien und Medizin Verlagsgesellschaft $\mathrm{mbH} \& \mathrm{Co}$. KG Neumarkter Straße 43 81637 München

ÄDA-Geschäftsstelle: Service Systems Carin Fresle, Ursula Raab E-Mail: aeda@sersys.de 